\title{
Change-Point Detection on Solar Panel Performance Using Thresholded LASSO
}

\author{
Youngjun Choe, ${ }^{\mathrm{a} * \dagger}$ Weihong Guo, ${ }^{\mathrm{b}}$ Eunshin Byon, ${ }^{\mathrm{a}}$ Jionghua (Judy) Jin ${ }^{\mathrm{a}}$ \\ and Jingjing $\mathrm{Li}^{\mathrm{c}}$
}

Solar energy is a fast growing energy source and has allowed the development of efficient, affordable, and easy-to-install photovoltaic systems over the years. Solar energy stakeholders are, however, concerned with sudden deterioration of photovoltaic systems' performance. Thus, effective change-point detection in solar panel performance analysis is essential for better harnessing solar energy and making photovoltaic systems more efficient. In particular, this study focuses on retrospectively identifying the time points of abrupt changes. Because the power generations from the solar panels are affected by a wide variety of factors, it is very difficult, if not impossible, to find a parametric model to detect abrupt changes in the power generation. We present a nonparametric detection method based on thresholded least absolute shrinkage and selection operator. The proposed method has low computational complexity and is able to accurately detect performance changes while being robust against false detection under noisy signals. The performance of the proposed method in detection of abrupt changes is evaluated and compared with state-of-the-art methods through extensive simulations and a case study using data collected from four solar energy facilities. We demonstrate that the proposed method is superior to benchmark methods. The proposed method will help solar energy stakeholders in several aspects including operations planning, maintenance scheduling, warranty underwriting, and cost-benefit analysis. Copyright $\odot 2016$ John Wiley \& Sons, Ltd.

Keywords: quality control; reliability; solar energy; time series

\section{Introduction}

$\mathrm{P}$ hotovoltaic systems harness the solar energy by directly converting solar radiation into electricity, with no noise, pollution or moving parts, making them reliable and long lasting. Over the past 10 years, U.S. solar energy installations have increased by a factor of $10 .^{1,2}$ The global market for solar energy is expected to triple by $2020 .^{3}$

Solar panels on the market typically come with 20 year warranties to guarantee that the panels will produce at least $80 \%$ of the rated power after 20 years of use. ${ }^{4}$ There are two aspects in analyzing the performance changes of the solar panels: gradual degradation and abrupt changes. Concerning the gradual degradation, the U.S. National Renewable Energy Laboratory conducted an extensive study and reported that the performance degradation patterns of solar panel systems depend on various factors such as technologies, ages, manufacturers, and geographic locations. ${ }^{5}$

Different from the gradual degradation, the performance of solar panels also experiences abrupt changes. For example, breakages of various components of solar panel systems are commonly observed in many solar energy facilities. Corrosion and thermal stresses result in the fracture of solar cells. Corrosion also causes the fracture of connectors and wires. The resulting sudden performance deterioration could significantly affect normal daily operations, maintenance scheduling, warranty underwriting, and financial analysis. As such, the detection of abrupt changes in solar panel health conditions becomes highly important to solar energy stakeholders. Despite its increasing importance, to the best of our knowledge, detection of abrupt changes in the solar panel system has not been studied well in the literature.

An example of the typical patterns of solar panel performance change is shown in Figure 1. This solar energy facility, located in Kaneohe, Hawaii, had eight $4 \times 6$ foot AC modules weighing 122 pounds each. The vertical axis in Figure 1 represents the daily average PV-to-POA ratio collected from August 1999 to November 2009, which is a commonly used health index in evaluating the performance of a solar panel. PV-to-POA ratio represents the solar panel's health condition and energy conversion efficiency, where ' $\mathrm{PV}$ ' denotes solar power output $(\mathrm{kW})$ and 'POA' denotes plane of array solar irradiance $\left(\right.$ watts $\left./ \mathrm{m}^{2}\right)$. Even after taking seasonality into consideration, we

\footnotetext{
${ }^{a}$ Department of Industrial and Operations Engineering, University of Michigan, 1205 Beal Avenue, Ann Arbor, MI 48109, USA

${ }^{b}$ Department of Industrial and Systems Engineering, Rutgers University, 96 Frelinghuysen Road, Piscataway, NJ 08854, USA

'Department of Mechanical Engineering, University of Hawai'i at Mānoa, 2540 Dole Street, Honolulu, HI 96822, USA

*Correspondence to: Youngjun Choe, Department of Industrial and Systems Engineering, University of Washington, 3900 NE Stevens Way, Seattle, WA 98195, USA.

${ }^{\dagger}$ E-mail:ychoe@uw.edu

${ }^{\ddagger}$ Harold and Inge Marcus Department of Industrial and Manufacturing Engineering, Pennsylvania State University, 310 Leonhard Building, University Park, PA 16802, USA
} 


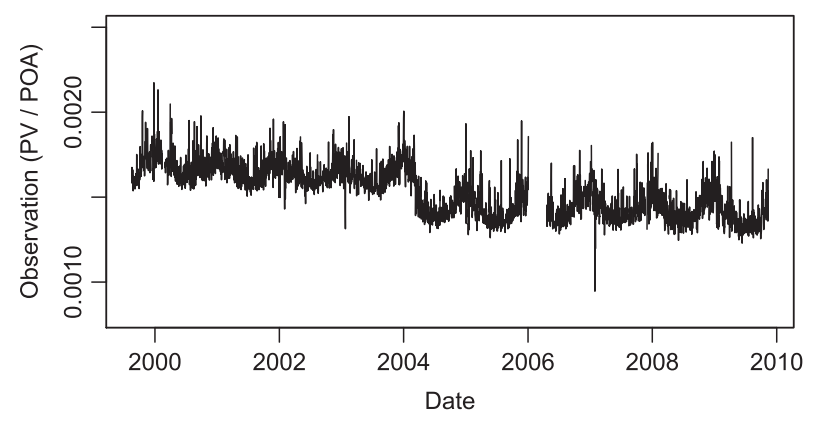

Figure 1. Observed PV-to-POA ratio for Facility D

can roughly observe that there is a significant sudden performance drop in the beginning of 2004. We note that in February 2004, the location experienced the largest quantity of precipitation in years, which was 9.49" of liquid precipitation, ${ }^{6}$ comparing with an average value of $2.01 .{ }^{7}$ The sudden drop in solar panel performance may be attributed to local or partial panel failure, possibly caused by the heavy precipitation. This example will be discussed further in Section 4.

There is considerable literature on detecting change points in general time series data, including online (sequential) detection of change points and off-line (retrospective) detection. Some applications are reasonable to assume that there is at most a single change point, ${ }^{8}$ whereas others need to consider multiple change points. ${ }^{9-11}$ In the literature on change-point detection, the definition of change point varies: Bai $^{9}$ considers shifts in multiple regression coefficients at certain times as change points; Roy et al. ${ }^{8}$ regard a change in the underlying network structure at a certain time based on a Markov random field model, but majority of the works consider changes (or jumps) in a time series of a response variable. Particularly, in many cases, detecting such changes can be reduced to identifying shifts in the mean of the time series. ${ }^{12,13}$

In our motivating example to be described in Section 4, we observe that the solar panels may have undergone multiple health condition changes during the data collection period. The solar panels are evaluated retrospectively for any abrupt performance changes. Therefore, in this study, we focus on the off-line detection of multiple change points in the mean of the time series of performance measurements. The off-line detection will be particularly useful for solar energy stakeholders in several aspects including operations planning, maintenance scheduling, warranty underwriting, and cost-benefit analysis.

The off-line detection of mean shifts has been most commonly formulated as multivariate optimization problems and then tackled by dynamic programming (DP). This approach utilizes the intrinsic additive nature of the least-square objective to recursively find the optimal change points. The major drawback of this approach is its computational complexity, ${ }^{10,14}$ which is typically of order $O\left(n^{2}\right)$, where $n$ is the number of observations. With the worst-case complexity of $O\left(n^{2}\right)$, a recently developed method called the pruned exact linear time (PELT) method ${ }^{11}$ is able to achieve the complexity of $O(n)$ if the number of change points increases at the same rate as $n$. However, this assumption is unreasonable for photovoltaic systems because the number of change points does not necessarily increase in proportion to $n$. In addition, despite its theoretical complexity of $O(n)$, PELT tends to be empirically slow in many practical settings. ${ }^{11}$ For off-line detection methods, the computational complexity has been of interest in the literature ${ }^{10,11,14}$ because detecting a few change points among a large number of time stamps is equivalent to finding the best solution among $2^{n-1}$ possible solutions.

Another widely used approach for off-line multiple change-point detection is binary segmentation (BS). BS first searches for a single change point from the entire dataset. If a change point is identified, the data are split into two subsegments at the change-point location. The single change-point detection procedure is then performed on either subsegment, possibly resulting in further splits. This process continues until no change points are found in any parts of the data. The computational complexity of $B S$ is $O(n$ log $n)$ and often empirically much faster than PELT and other DP-based approaches. However, because each stage of BS involves the search for a single change point, BS tends to be less accurate in change-point estimation than other methods, ${ }^{11,14}$ especially when multiple change points are contained. Fryzlewicz ${ }^{14}$ shows that BS is only consistent in estimating the number and locations of multiple change points when the minimum spacing between any two adjacent change points is of order greater than $n^{3 / 4}$, which is relatively large and may not be satisfied in solar panel performance changes. Wild BS (WBS), ${ }^{14}$ a recently developed method, improves BS for the consistent estimation.

A different route to tackling the multiple change-point detection problem is taken by Harchaoui and Lévy-Leduc ${ }^{10,15}$ who propose to use the least absolute shrinkage and selection operator (LASSO) for the off-line detection of mean shifts. The benefits of this approach include low computational complexity of at most $O(n \log (n))$ and capability in handling large datasets. On the other hand, LASSO tends to choose more change points than necessary. ${ }^{15} \mathrm{~A}$ remedy proposed by Harchaoui and Lévy-Leduc ${ }^{10}$ is to add a reduced DP to the final step in order to remove potentially false change points. They acknowledge, however, that the addition of reduced DP is a heuristic approach and lacks thorough theoretical support.

The objective of this article is to develop a LASSO-based nonparametric approach that is accurate and robust in detecting abrupt performance changes of solar panel array. We capitalize the benefits of LASSO but remedy its false detection problem by using the thresholded LASSO (TLASSO) proposed in the study of Zhou. ${ }^{16}$ TLASSO was originally developed to enhance LASSO by reducing the number of false positive variable selections in a high-dimensional linear model. However, inspired by Harchaoui and Lévy-Leduc, ${ }^{10}$ we note that our change-point detection problem can be formulated as a variable selection problem, and we utilize the benefits of TLASSO to reduce falsely detected change points. TLASSO maintains the same level of computational complexity of LASSO (i.e., at most $O(n \log (n))$ and empirically takes the similar computational time with other fast methods of $O(n \log (n))$. To the best of our knowledge, our work is the first study that employs TLASSO in the context of change-point detection. Our preliminary study ${ }^{17}$ explored 
the feasibility of using TLASSO for off-line multiple change-point detection. This article further explores the accuracy and robustness of TLASSO-based method for detecting abrupt changes and demonstrates its benefit over two competing state-of-the-art methods, WBS and LASSO with reduced DP. Results from simulations and solar panel degradation analysis suggest that the TLASSO approach is able to accurately detect not only performance changes but also robust under many uncertainties.

This article is organized as follows. In Section 2, we present the TLASSO method with backgrounds on problem formulation, LASSO-based detection methods, and WBS. In Section 3, TLASSO and state-of-the-art methods are compared via simulation studies. In Section 4, we exhibit the performance of the proposed method in a case study with data collected from four solar energy facilities. Finally, we provide our conclusions and summarize our future research directions.

\section{Methods for off-line change-point detection}

In this section, we present four methods that can be used for off-line detection of multiple change points. Specifically, we will introduce the proposed TLASSO-based method and review three benchmark methods, namely, LASSO, LASSO with reduced DP, and WBS.

First, the mathematical formulation of the off-line detection of mean shifts is described as follows. Suppose that there are $K$ change points, namely, $\tau_{1}, \ldots, \tau_{K}$, where $K$ is unknown. Consider the following piecewise constant model:

$$
Y_{t}=\mu_{k}+\epsilon_{t}
$$

where $\tau_{k-1} \leq t \leq \tau_{k}-1, k=1, \ldots, K+1, t=1, \ldots, n$ with $\tau_{0}=1$ and $\tau_{K+1}=n+1$. The response variable, $Y_{t}$, denotes the PVto-POA ratio at time $t$. The noises, $\left\{\epsilon_{t}\right\}_{1 \leq t \leq n}$, are i.i.d. zero-mean random variables with finite variance, $\sigma^{2}$. With this model, our goal is to estimate the model parameters, $K, \tau_{1}, \ldots, \tau_{K}$, and $\mu_{1}, \ldots, \mu_{K+1}$, that can best explain the observations, $\left\{Y_{t}\right\}_{1 \leq t \leq n}$. Note that no constraints are imposed on $\left\{\mu_{k}\right\}_{1 \leq k \leq k+1}$, although the performance changes may have a certain direction, for example, $\mu_{k+1}-\mu_{k} \leq 0$ for $k=1, \ldots, K$.

\subsection{LASSO-based change-point detection}

Model parameters, $\tau_{1}, \ldots, \tau_{K}$ and $\mu_{1}, \ldots, \mu_{K+1}$, can be estimated by minimizing

$$
\sum_{k=1}^{K+1} \mathcal{C}\left(Y_{\tau_{k-1}, \ldots, Y_{\tau_{k}-1}}\right)+\lambda f(K)
$$

where $\mathcal{C}$ is a cost function for a segment and $\lambda f(K)$ is a penalty to the model complexity. ${ }^{11}$ The LASSO-based change-point detection method proposed by Harchaoui and Lévy-Leduc ${ }^{10}$ considers a squared loss function of $\mathcal{C}$ and penalizes the total variation as follows:

$$
\min _{\mathbf{u} \in \mathbb{R}^{n}} \frac{1}{n} \sum_{t=1}^{n}\left(Y_{t}-u_{t}\right)^{2}+\lambda_{n} \sum_{t=1}^{n-1}\left|u_{t+1}-u_{t}\right| .
$$

Harchaoui and Lévy-Leduc ${ }^{10}$ show that this change-point detection problem can be cast into an equivalent variable selection problem by setting $\mathbf{u} \equiv \mathbf{X}_{n} \boldsymbol{\beta}$ :

$$
\min _{\boldsymbol{\beta} \in \mathbb{R}^{n}} \frac{1}{n} \sum_{t=1}^{n}\left(Y_{t}-\left(\mathbf{X}_{n} \boldsymbol{\beta}\right)_{t}\right)^{2}+\lambda_{n} \sum_{t=2}^{n}\left|\beta_{t}\right|,
$$

where $\mathbf{X}_{n}$ is the $n \times n$ lower triangular matrix with all nonzero elements being ones. That is, all the entries above the main diagonal are zeros and the rest of the entries are ones. Then, nonzero $\beta_{t}, t=2, \ldots, n$ encodes the jump size and direction at the estimated change point of $t$. The formulation in (4) has an important implication because finding the change points becomes a variable selection problem for which LASSO provides a path of solutions over different $\lambda_{n}$ very efficiently. ${ }^{18}$

A drawback of the LASSO-based detection is that it tends to choose more change points than the true number. ${ }^{10}$ As an illustrative example, let us consider $K=2$ with $\mu_{1}=0, \mu_{2}=1, \mu_{3}=0.5, \epsilon_{t} \stackrel{i i d}{\sim} N(\mu=0, \sigma=0.3), t=1, \ldots, n$, in (1), where the change points are located at $\tau_{1}=1001$ and $\tau_{2}=2001$. The LASSO regularization path in Figure 2(a) can be interpreted as follows: the saturated model that regards all time stamps as change points explains $100 \%$ of deviance (i.e., all variation in the observed responses), while the null model that assumes the absence of change points explains $0 \%$ of deviance. For example, over $60 \%$ of deviance can be explained with four change points chosen. However, among the four change points, some have fairly small coefficients, indicating that we could prune them to avoid false positives. Figure 2 (b) visualizes the observations from the given data generating model and the estimated change points.

We also summarize the limitations of the LASSO-based change-point detection method in the theoretical point of view. First, as shown in the study of Harchaoui and Lévy-Leduc, ${ }^{10} \mathbf{C}_{n}=n^{-1} \mathbf{X}_{n}^{\top} \mathbf{X}_{n}$ does not satisfy the irrepresentable condition, ${ }^{19}$ implying that a perfect estimation of the change points is not possible. They also proved that for $m_{n}=s_{n} \log n$, with $s_{n}$ being the sparsity (i.e., the number of nonzero coefficients), $\phi_{\min }\left(m_{n}\right) \leq 1 / n$ holds for all $n \geq 1$, where $\phi_{\min }(m)$ is the $m$-sparse minimal eigenvalue of $\boldsymbol{C}_{n}$ as defined in the study of Meinshausen, ${ }^{20}$ if all true change points are adjacent to each other. This implies that $\mathbf{C}_{n}$ does not satisfy the incoherent design condition that ensures $I_{2}$-consistency, while $s_{n}$ is allowed to grow almost as fast as the sample size. Accordingly, Harchaoui 


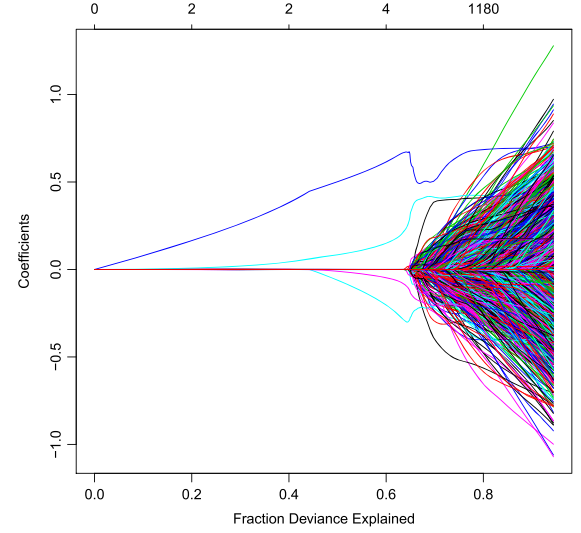

(a) Regularization paths for LASSO: Top $x$-axis denotes the degree of freedom (or the number of change-points chosen)

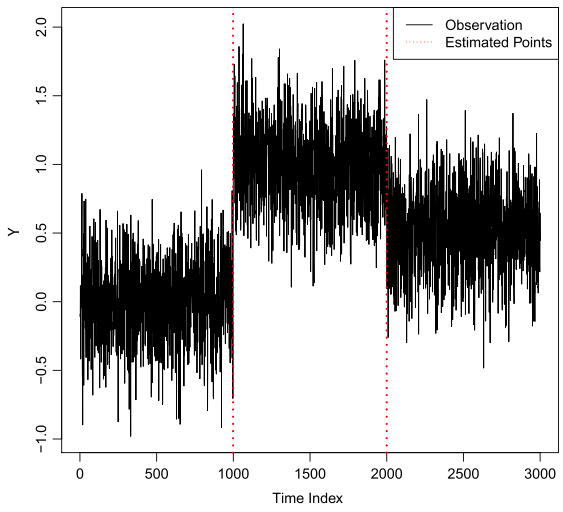

(b) LASSO-based detection: four change-points at Time Index $=999,1000,2000$, and 2001

Figure 2. Illustration of least absolute shrinkage and selection operator (LASSO)-based change-point detection

and Lévy-Leduc ${ }^{10}$ limit the maximum number of change points (i.e., bound the sparsity from above by a constant) to establish $I_{2}-$ consistency. Furthermore, they establish the consistent estimation of change points, $\tau_{1}, \ldots, \tau_{k}$, in (1) by (a) assuming that $\epsilon_{1}, \ldots, \epsilon_{n}$ are iid with a sub-Gaussian distribution, (b) bounding the minimum interval between change points, $I_{\min }=\min _{1 \leq k \leq K}\left|\tau_{k+1}-\tau_{k}\right|$, from below, and (c) bounding the minimum jump size, $J_{\min }=\min _{1 \leq k \leq K}\left|\mu_{k+1}-\mu_{k}\right|$, from below.

With these theoretical properties, for large $n$, change points estimated by LASSO will include most (if not all) true change points because they will be correctly identified with a high probability (see Proposition 3 in the study of Harchaoui and Lévy-Leduc ${ }^{10}$ ). Moreover, we will only see a small number of false positives (i.e., $\left\{t \in\{1, \ldots, n\}: \beta_{t}=0, \hat{\beta}_{t} \neq 0\right\}$ ) with small coefficients when $n$ is large (see Proposition 2 in the study of Harchaou and Lévy-Leduc ${ }^{10}$ ). Therefore, we believe that an appropriate pruning based on coefficient sizes can help reduce false positives while keeping the correctly estimated change points, which motivates the use of TLASSO.

\subsection{Least absolute shrinkage and selection operator with reduced dynamic programming-based pruning}

Before presenting the proposed change-point detection method with TLASSO, we discuss another pruning method based on the reduced DP (rDP), proposed by Harchaoui and Lévy-Leduc ${ }^{15}$ to find a good subset of the change points identified by the LASSO-based detection. Let $S=\left\{\hat{\tau}_{1}, \ldots, \hat{\tau}_{K_{\max }}\right\}$ denote the set of change points estimated by LASSO. Then, rDP computes the minimum loss for choosing $\hat{K}$ change points as follows:

$$
J(\hat{K})=\min _{\substack{\eta_{1}<\cdots<\eta_{\hat{K}} \\ \text { s.t. } \eta_{1}, \cdots, \eta_{\hat{K}} \in S}} \sum_{k=1}^{\hat{K}+1} \sum_{t=n_{k-1}}^{\eta_{k}-1}\left(Y_{t}-\hat{\mu}_{k}\right)^{2}
$$

for $\hat{K}$ in $\left\{1, \ldots, K_{\max }\right\}$ with $\eta_{0}=1$ and $\eta_{\hat{K}+1}=n+1$.

The rule of thumb suggested in the study of Harchaoui and Lévy-Leduc ${ }^{15}$ decides the final number of change points, $K^{*}=$ $\min _{k \geq 1}\{J(k+1) / J(k) \geq 1-v\}$, using a threshold, $v$. This threshold is set as 0.05 or 0.01 , in the study of Harchaoui and Lévy-Leduc. ${ }^{15}$ We call this approach LASSO+rDP. This approach appears to be effective in practice but does not have thorough theoretical support. ${ }^{10}$

\subsection{Thresholded least absolute shrinkage and selection operator for change-point detection}

In this article, we propose to use TLASSO ${ }^{16}$ for detecting abrupt changes of solar panel performance. As mentioned earlier, TLASSO was originally designed to improve LASSO in estimating the parameters of a high-dimensional linear model. TLASSO maintains the similar prediction and estimation error with LASSO while substantially reducing the false positives in variable selection. ${ }^{21}$ TLASSO has not been used for the change-point detection, but we note that TLASSO can benefit the change-point detection by better protecting against false positives than the existing methods that do not focus on reducing false positives.

We apply TLASSO to the change-point detection problem that is cast into the variable selection formulation in (4). We assume that $\beta_{\min }:=\min \left\{\left|\beta_{t}\right|: \beta_{t} \neq 0, t \in\{1, \ldots, n\}\right\}$ is sufficiently large because we want to detect large changes of PV-to-POA ratios, not small noises that are of little practical interests. Following the implementation procedure theoretically supported by Zhou, ${ }^{16}$ we obtain an intial estimator $\hat{\boldsymbol{\beta}}_{\text {init }}=\left(\hat{\beta}_{1, \text { init }}, \cdots, \hat{\beta}_{n, \text { init }}\right)^{T}$ from LASSO solving (4) and recommend using the parameter, $\lambda_{n}=\lambda \sigma$, where $\lambda$ is $\sqrt{2(\log n) / n}$ and $\sigma$ is estimated if unknown. Then, we take the following procedure:

\section{TLASSO procedure}

1. First Thresholding: Threshold $\hat{\boldsymbol{\beta}}_{\text {init }}$ to obtain 


$$
I=\left\{t \in\{1, \ldots, n\}:\left|\hat{\beta}_{t, \text { init }}\right| \geq \xi_{0}\right\}
$$

where the threshold, $\xi_{0}$, is set as $\lambda \sigma$.

2. Refitting: Refit the data with the ordinary least squares, $\hat{\beta}_{l}=\left(\mathbf{X}_{l}^{T} \mathbf{X}_{l}\right)^{-1} \mathbf{X}_{l}^{T} \mathbf{Y}$ and $\hat{\beta}_{l c}=0$, where $\mathbf{X}_{l}$ is the $n \times|I|$ submatrix consisting of the columns of $\mathbf{X}_{n}$, indexed by $l$; similarly, $\hat{\beta}_{l}$ is a subvector of $\hat{\beta}$ confined to $l$.

3. Second Thresholding: Threshold $\hat{\beta}_{l}$ with $\xi_{1}=4 \lambda_{n} \sqrt{|I|}$ to obtain

$$
J=\left\{t \in I:\left|\hat{\beta}_{t}\right| \geq \xi_{1}\right\} \subseteq l .
$$

4. Final Fitting: Conduct Step 2 with $J$ in place of $/$ to obtain the final estimates, $\hat{\beta}_{J}$ and $\hat{\beta}_{\jmath}$. The set, $J$, denotes the time points of abrupt changes.

As we can see in Steps 1 and 3, TLASSO sets small nonzero coefficients to zeros through twice of thresholding in order to prune potential false positives according to the rationale explained at the end of Section 2.1. The TLASSO procedure including the thresholds, $\xi_{0}$ and $\xi_{1}$, is theoretically proven to yield accurate identification of the true nonzero coefficients in the linear model. ${ }^{16}$ This implies that the given thresholding rules provide accurate detection of true change points in our problem. In particular, different from LASSO+rDP, TLASSO is known to have desirable theoretical properties such as consistent estimation of $\boldsymbol{\beta}_{,}{ }^{16}$ that is, consistent estimation of change points. We also note that the solution from TLASSO does not necessarily reside in the solution path of LASSO, implying that varying the penalty parameter for LASSO would not necessarily give us the same solution from TLASSO.

When we need to estimate $\sigma$, we can use the standard deviation of observed noise as the maximum likelihood estimator under the assumption of Gaussian noises. ${ }^{22}$ We, however, note that the 'observed noise' calculated under a wrong model (e.g., assuming no change point when there actually exist change points) can result in a biased estimator of $\sigma$. For our case study where responses mainly show trend and seasonality, we calculate the standard deviation of noise after removing trend and seasonality from the observed response values. For more complicated datasets, future research may explore an iterative approach, for example, the estimate of $\sigma$ is refined to consider the possible change points in each iteration.

Because the number of abrupt changes we aim to detect in solar panel performance would be small compared with the total number of time stamps, $n$, TLASSO procedure keeps the same computational complexity of LASSO-only approach, namely, at most $O(n$ log $(n))$. Moreover, TLASSO will help reduce false positives while keeping the correctly estimated change points, allowing engineers to focus on a few critical changes in solar panel performance.

It is known that TLASSO performs similarly with another famous variation of LASSO, adaptive LASSO, ${ }^{23}$ in terms of prediction and estimation. ${ }^{21}$ Both TLASSO and adaptive LASSO ${ }^{23}$ enhance LASSO by reducing false positives, but the upper bound on the number of false positives of TLASSO is tighter than that of adaptive LASSO, ${ }^{21}$ leading us to favor TLASSO in order to potentially better guard against false positives. Also, TLASSO requires less stricter condition on $\beta_{\min }$ than adaptive LASSO to achieve exact change-point estimation, ${ }^{21}$ making TLASSO a better choice for detecting solar panel performance changes that are subject to various sizes of stochastic noises.

\subsection{Wild binary segmentation for change-point detection}

The WBS ${ }^{14}$ method for multiple change-point detection has been demonstrated to be computationally fast and perform very well in many applications. Considering WBS as a benchmark, we will compare TLASSO-based detection with WBS in simulation and case studies. This subsection briefly reviews the WBS method.

The basic element of WBS is the cumulative sum (CUSUM) statistic defined as

$$
\tilde{Y}_{s, e}^{b}=\sqrt{\frac{e-b}{(e-s+1)(b-s+1)}} \sum_{i=s}^{b} Y_{i}-\sqrt{\frac{b-s+1}{(e-s+1)(e-b)}} \sum_{i=b+1}^{e} Y_{i},
$$

where $s \leq b<e$. The first step of WBS computes $\tilde{Y}_{1, n}^{b}$ and then takes $b_{1,1}=\arg \max _{b: 1 \leq b<n}\left|\tilde{Y}_{1, n}^{b}\right|+1$ to be the first change-point candidate. If $b_{1,1}$ is judged to be significant (based on a certain threshold $\zeta_{n}$, which is compared with the CUSUM statistic), the domain $[1, n]$ is split into two subintervals to the left and right of $b_{1,1}$ and the recursion continues by computing $\tilde{Y}_{1, b_{1,1}-1}^{b}$ and $\tilde{Y}_{b_{1,1}, n^{\prime}}^{b}$ possibly resulting in further splits. Instead of using fixed intervals in the standard BS algorithm, WBS randomly draws a number of pairs ( $s, e$ ) and compute the CUSUM statistic, $\tilde{Y}_{s, e}^{b}$.

Noting that the number of estimated change points is a nonincreasing function of the threshold, $\zeta_{n}$, Fryzlewicz ${ }^{14}$ also proposes a way to circumvent the need to choose $\zeta_{n}$ by directly selecting the number of change points. This approach minimizes a new model selection criterion called 'strengthened Schwarz Information Criterion' (sSIC), which is defined as

$$
\operatorname{sSIC}(k)=\frac{n}{2} \log \hat{\sigma}_{k}^{2}+k(\log n)^{\alpha}
$$

where $k$ is the number of change points, $\hat{\sigma}_{k}^{2}$ is the corresponding maximum likelihood estimator of the residual variance under Gaussian noise assumption, and $\alpha>1$ is a constant parameter. The standard SIC penalty corresponds to the choice of $\alpha=1$, thus $\alpha>1$ is required in order to result in a stronger penalty than the standard SIC. Fryzlewicz ${ }^{14}$ recommends $\alpha=1.01$. 


\section{Performance evaluation using simulation study}

In this section, we conduct simulations to evaluate and compare the performances of the change-point detection methods. Each of the four detection methods, WBS, LASSO, LASSO+rDP, and TLASSO, depends on some tuning parameter (e.g., threshold or regularization parameter) that determines the final number of change-point estimates. Because of the importance of the tuning parameter, we perform simulation studies to investigate the robustness of detection performance as we vary the tuning parameter.

The tuning parameter in each method has a monotonic relationship with the number of estimated change points, $\hat{K}$. We start with a tuning parameter that gives much larger $\hat{K}$ than the true number of change points, $K$, and varies the tuning parameter to incrementally decrease $\hat{K}$ down to one. To this end, for WBS, we increment threshold, $\zeta_{n}$, to yield a smaller $\hat{K}$. For LASSO, we increase the regularization parameter and select $\hat{\beta}$ that explains the largest deviance for each $\hat{K}$. For LASSO+rDP, a larger threshold, $v$, leads to a smaller $\hat{K}$. For TLASSO, we obtain $\hat{\boldsymbol{\beta}}_{\text {init }}$ from LASSO with $0.1 \lambda_{n}$ for $\lambda_{n}$ defined in Section 2 and then threshold $\hat{\boldsymbol{\beta}}_{\text {init }}$ as in the first step of the TLASSO procedure in Section 2 . We increment the threshold $\xi_{0}$ to obtain a smaller $\hat{K}$.

Change-point detection error can be measured as a difference between two sets, the set of true change points and the set of estimated change points. Boysen et al. ${ }^{24}$ define the following set difference measure for two sets, $A$ and $B$, by

$$
\mathcal{E}(A \| B)=\sup _{b \in B} \inf _{a \in A}|a-b| .
$$

Harchaoui and Lévy-Leduc ${ }^{10}$ use this measure to quantify the two types of detection error. First, false positive measure (FPM) is $\mathcal{E}(\mathcal{T} \| \hat{\mathcal{T}})$, where $\mathcal{T}=\left\{\tau_{k}, k=1, \ldots, K\right\}$ is the set of true change points and $\hat{\mathcal{T}}=\left\{\hat{\tau}_{k}, k=1, \ldots, \hat{K}\right\}$ is the set of estimated change points. Next, false negative measure (FNM) is $\mathcal{E}(\hat{\mathcal{T}} \| \mathcal{T})$. The larger one between FPM and FNM is called the Hausdorff distance between $\mathcal{T}$ and $\hat{\mathcal{T}}$

$$
\Delta(\mathcal{T}, \hat{\mathcal{T}})=\sup \{\mathcal{E}(\mathcal{T} \| \hat{\mathcal{T}}), \mathcal{E}(\hat{\mathcal{T}} \| \mathcal{T})\}
$$

Therefore, perfect change-point detection is equivalent to zero Hausdorff distance.

For simulation study, we consider two data generating models. The first model takes the same pattern of stairs 10 model in the study of Fryzlewicz ${ }^{14}$ but reverses the shift directions from upwards to downwards so that we can model the performance drops. We use the same number of change points, $K=14$, and set $n=1000$ as in the study of Fryzlewicz. ${ }^{14}$ Figure 3 shows the typical datasets generated from the model with several noise levels.

Figure 4 shows the average FPM and FNM based on 100 replications for the four detection methods with three noise levels. The horizontal axis denotes $\hat{K}$, and the vertical axis represents the error measures, FPM and FNM. From all plots, we observe larger FPM (smaller FNM) as more change points (i.e., larger $\hat{K}$ ) are required to estimate. Ideally, when $\hat{K}$ is equal to the true number of change points, $K=14$, we hope the Hausdorff distance (i.e., the larger one between FPM and FNM) to be close to zero. LASSO detection errors reported in the second row of Figure 4 show that LASSO does not achieve this goal in this example. On the other hand, WBS (in the first row), LASSO+rDP (in the third row), and TLASSO (in the fourth row) maintain the Hausdorff distance close to zero for $\hat{K}=14$. WBS performs similarly with LASSO+rDP. Both of them generally have lower FNM than TLASSO when $\hat{K}<K$. That is, some true change points missed by TLASSO would be farther from the estimated change points than other methods in this example (this pattern reverses in the next data generating model that is more complicated than this model). On the other hand, when $\hat{K}>K$, TLASSO maintains lower FPM than other methods, showing the robustness against the false positives. Therefore, false positives from TLASSO would be closer to true change points than those from other methods.

The second data generating model is the Blocks model in the study of Donoho and Johnstone. ${ }^{25}$ This model is more complicated than the first model and is known as a difficult model for change-point detection because of large heterogeneity. ${ }^{10}$ The spaces between $K=11$ change points are irregular, and the shift directions can be both upwards and downwards. Figure 5 shows the typical datasets with different noise levels. We again set $n=1000$ and average FPM and FNM over 100 replications.

Figure 6 shows the detection errors for the four methods with three noise levels. We observe generally similar findings with the first data generating model. LASSO in the second row does not achieve zero Hausdorff distance at $\hat{K}=K=11$ for all three noise levels. WBS and LASSO+rDP show similar performance. For $\hat{K}<K$, TLASSO tends to have smaller or similar FNM than WBS and LASSO+rDP. For $\hat{K}>K$, TLASSO shows a distinctly better FPM pattern than WBS and LASSO+rDP, echoing the observation from the first data generating model. The results suggest that TLASSO would be more robust against false positives.

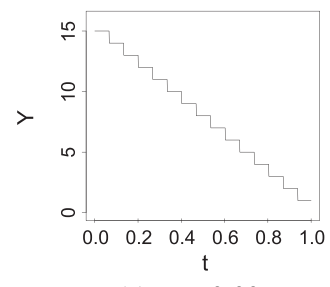

(a) $\sigma=0.00$

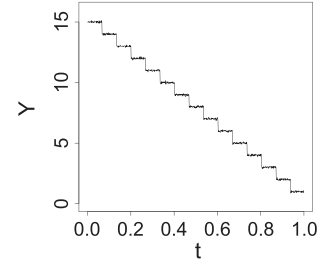

(b) $\sigma=0.05$

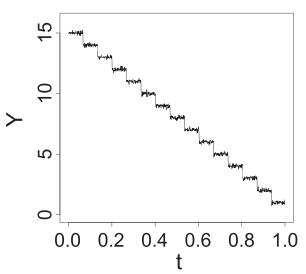

(c) $\sigma=0.10$

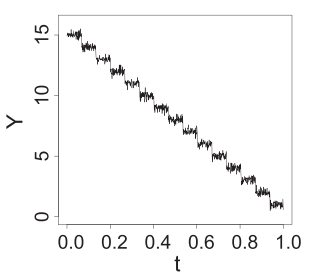

(d) $\sigma=0.20$

Figure 3. Stairs example with different noise levels 

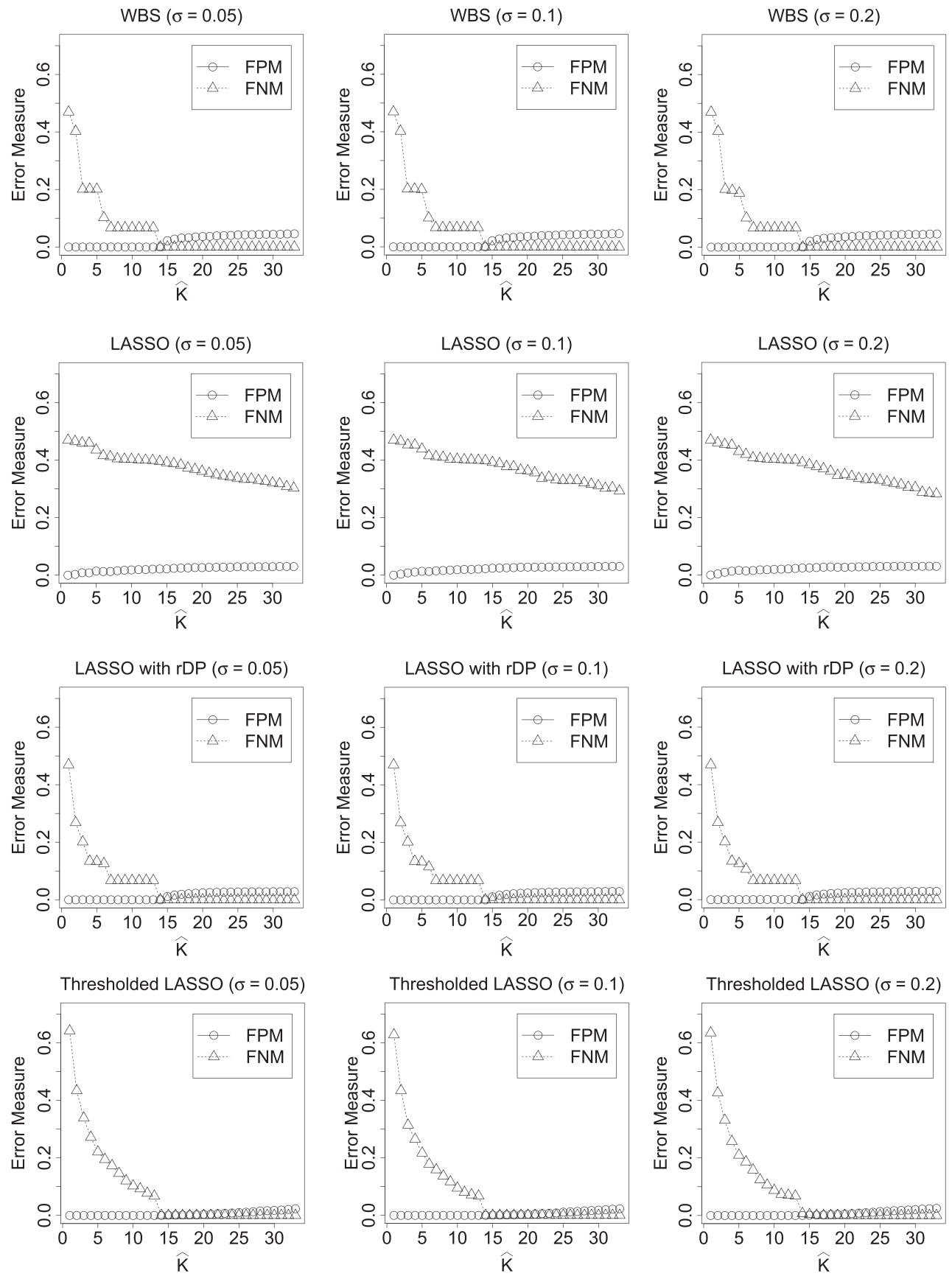

Figure 4. Comparison of WBS (first row), LASSO (second row), LASSO+rDP (third row), and TLASSO (fourth row) for the first data generating model with three different noise levels, $\sigma=0.05,0.10$, and 0.20 , from left to right. FNM, false negative measure; FPM, false positive measure; LASSO+rDP, least absolute shrinkage and selection operator with reduced dynamic programming; TLASSO, thresholded least absolute shrinkage and selection operator; WBS, wild binary segmentation

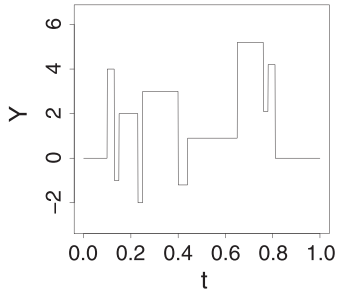

(a) $\sigma=0.00$

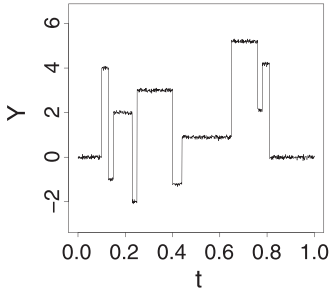

(b) $\sigma=0.05$

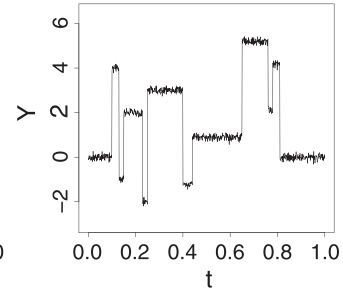

(c) $\sigma=0.10$

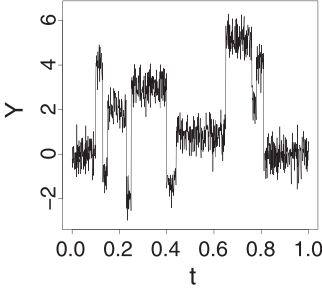

(d) $\sigma=0.50$

Figure 5. Blocks example with different noise levels 

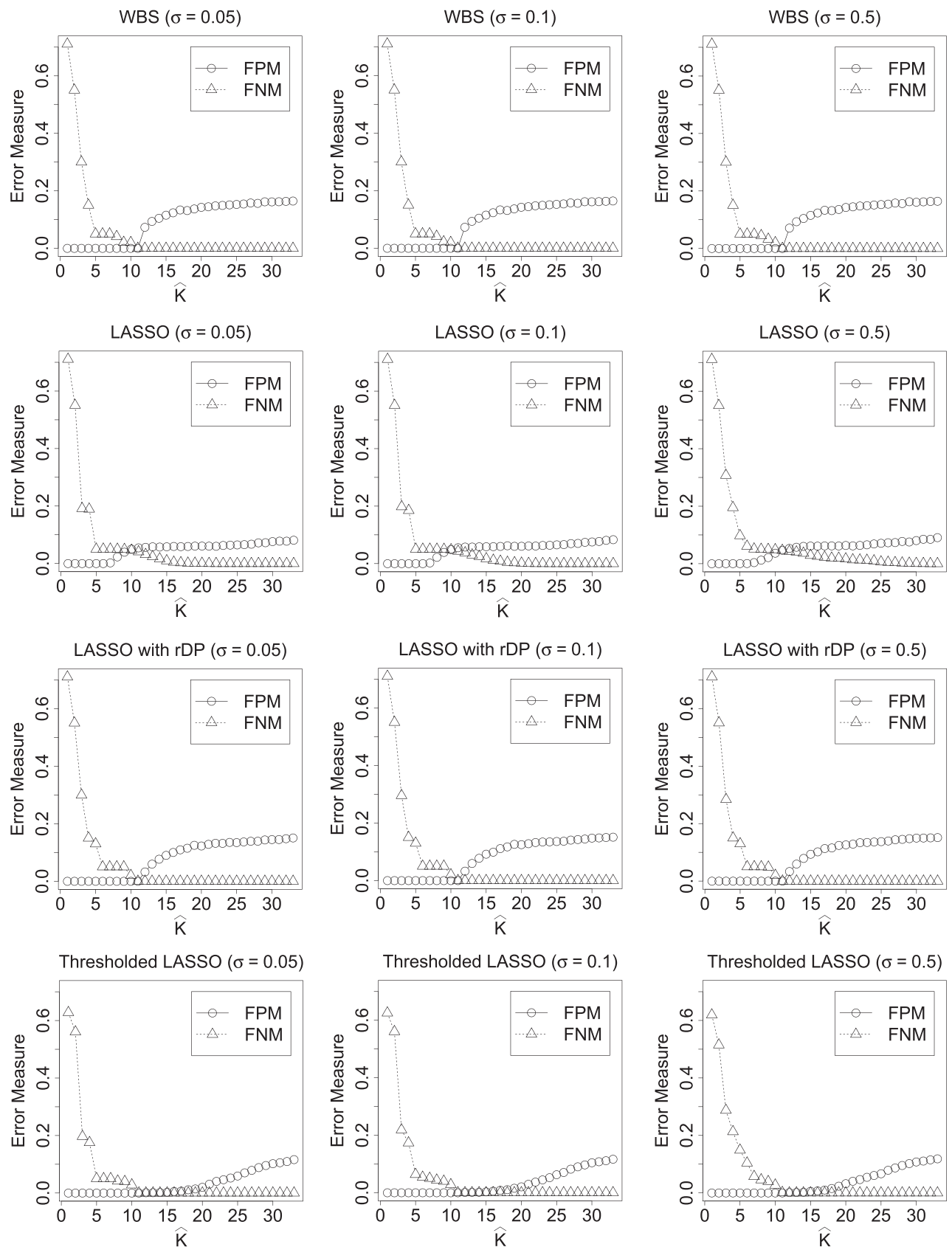

Figure 6. Comparison of WBS (first row), LASSO (second row), LASSO+rDP (third row), and TLASSO (fourth row) for the second data generating model with three different noise levels, $\sigma=0.05,0.10$, and 0.50 , from left to right. FNM, false negative measure; FPM, false positive measure; LASSO+rDP, least absolute shrinkage and selection operator with reduced dynamic programming; TLASSO, thresholded least absolute shrinkage and selection operator; WBS, wild binary segmentation

We now compare the performances of WBS, LASSO+rDP, and TLASSO under their recommended parameter settings, which include sSIC with $\alpha=1.01$ for WBS according to Fryzlewicz, ${ }^{14} v=0.05$ for LASSO+rDP according to Harchaoui and Lévy-Leduc, ${ }^{15}$ and $\lambda_{n}=\lambda \sigma$ for TLASSO. Note that we omit LASSO because no guideline for this detection method was provided in the literature. Table I shows the results based on 100 replications for the first data generating model. $\hat{K}$ is the number of estimated change points. Although it is generally better for $\hat{K}$ to be close to the true number of change points, $K=14, \hat{K}$ being equal to 14 does not mean that the locations of the true change points are correctly identified. To evaluate the actual detection performance, we need to consider FPM and FNM. LASSO+rDP underestimates the number of change points for the three noise sizes $(\sigma=0.05,0.10$, and 0.20$)$, resulting in relatively large FNMs compared with other two methods. For small noise sizes (i.e., $\sigma=0.05$ and 0.10 ), TLASSO and WBS yield similar $\hat{K}$ and the same FNMs, but TLASSO leads to smaller FPMs on average with smaller standard deviations. This robustness against false positives echoes the observation from Figure 4. When the noise size is large (i.e., $\sigma=0.20$ ), the recommended setting for TLASSO results in a smaller $\hat{K}$ and a larger FNM than WBS, even though TLASSO's FPM stays very small. This conservative estimation result can be understood from the fact that TLASSO assumes the sufficiently large $\beta_{\text {min }}$ to focus on large changes, not small jumps comparable with noises (cf. Section 2.3). 


\begin{tabular}{|c|c|c|c|c|c|c|c|c|c|}
\hline \multirow[b]{2}{*}{$\sigma$} & \multicolumn{3}{|c|}{$\hat{K}$} & \multicolumn{3}{|c|}{ FPM } & \multicolumn{3}{|c|}{ FNM } \\
\hline & 0.05 & 0.10 & 0.20 & 0.05 & 0.10 & 0.20 & 0.05 & 0.10 & 0.20 \\
\hline WBS & $\begin{array}{l}14.05 \\
(0.22)\end{array}$ & $\begin{array}{l}14.07 \\
(0.26)\end{array}$ & $\begin{array}{l}14.10 \\
(0.36)\end{array}$ & $\begin{array}{c}0.00076 \\
(0.00363)\end{array}$ & $\begin{array}{c}0.00078 \\
(0.00363)\end{array}$ & $\begin{array}{c}0.00111 \\
(0.00361)\end{array}$ & $\begin{array}{c}0.00000 \\
(0.00000)\end{array}$ & $\begin{array}{c}0.00000 \\
(0.00000)\end{array}$ & $\begin{array}{c}0.00029 \\
(0.00048)\end{array}$ \\
\hline LASSO+rDP & $\begin{array}{c}7.42 \\
(0.91)\end{array}$ & $\begin{array}{c}6.60 \\
(0.85)\end{array}$ & $\begin{array}{c}5.80 \\
(0.88)\end{array}$ & $\begin{array}{c}0.00000 \\
(0.00000)\end{array}$ & $\begin{array}{c}0.00001 \\
(0.00010)\end{array}$ & $\begin{array}{c}0.00035 \\
(0.00058)\end{array}$ & $\begin{array}{c}0.07579 \\
(0.02267)\end{array}$ & $\begin{array}{c}0.08786 \\
(0.03117)\end{array}$ & $\begin{array}{c}0.10725 \\
(0.03295)\end{array}$ \\
\hline TLASSO & $\begin{array}{l}14.23 \\
(0.45)\end{array}$ & $\begin{array}{l}14.20 \\
(0.43)\end{array}$ & $\begin{array}{l}11.08 \\
(1.71)\end{array}$ & $\begin{array}{c}0.00022 \\
(0.00042)\end{array}$ & $\begin{array}{c}0.00021 \\
(0.00046)\end{array}$ & $\begin{array}{c}0.00006 \\
(0.00024)\end{array}$ & $\begin{array}{c}0.00000 \\
(0.00000)\end{array}$ & $\begin{array}{c}0.00000 \\
(0.00000)\end{array}$ & $\begin{array}{c}0.07580 \\
(0.03647)\end{array}$ \\
\hline
\end{tabular}

Note: Each cell contains the average in the first line and the standard deviation in parentheses in the second line based on 100 replications. FNM, false negative measure; FPM, false positive measure; LASSO+rDP, least absolute shrinkage and selection operator with reduced dynamic programming; TLASSO, thresholded least absolute shrinkage and selection operator; WBS, wild binary segmentation.

\begin{tabular}{|c|c|c|c|c|c|c|c|c|c|}
\hline \multirow[b]{2}{*}{$\sigma$} & \multicolumn{3}{|c|}{$\hat{K}$} & \multicolumn{3}{|c|}{ FPM } & \multicolumn{3}{|c|}{ FNM } \\
\hline & 0.05 & 0.10 & 0.50 & 0.05 & 0.10 & 0.50 & 0.05 & 0.10 & 0.50 \\
\hline WBS & $\begin{array}{l}11.08 \\
(0.31)\end{array}$ & $\begin{array}{l}11.08 \\
(0.31)\end{array}$ & $\begin{array}{l}11.11 \\
(0.37)\end{array}$ & $\begin{array}{c}0.00402 \\
(0.02136)\end{array}$ & $\begin{array}{c}0.00402 \\
(0.02136)\end{array}$ & $\begin{array}{c}0.00409 \\
(0.02134)\end{array}$ & $\begin{array}{c}0.00000 \\
(0.00000)\end{array}$ & $\begin{array}{c}0.00000 \\
(0.00000)\end{array}$ & $\begin{array}{c}0.00005 \\
(0.00022)\end{array}$ \\
\hline LASSO+rDP & $\begin{array}{c}6.09 \\
(0.92)\end{array}$ & $\begin{array}{c}6.86 \\
(0.94)\end{array}$ & $\begin{array}{c}7.40 \\
(1.20)\end{array}$ & $\begin{array}{c}0.00000 \\
(0.00000)\end{array}$ & $\begin{array}{c}0.00000 \\
(0.00000)\end{array}$ & $\begin{array}{c}0.00028 \\
(0.00241)\end{array}$ & $\begin{array}{c}0.07087 \\
(0.03530)\end{array}$ & $\begin{array}{c}0.05375 \\
(0.01786)\end{array}$ & $\begin{array}{c}0.05056 \\
(0.01649)\end{array}$ \\
\hline TLASSO & $\begin{array}{l}11.34 \\
(0.57)\end{array}$ & $\begin{array}{l}11.27 \\
(0.49)\end{array}$ & $\begin{array}{c}6.45 \\
(0.59)\end{array}$ & $\begin{array}{c}0.00029 \\
(0.00046)\end{array}$ & $\begin{array}{c}0.00025 \\
(0.00044)\end{array}$ & $\begin{array}{c}0.00081 \\
(0.00160)\end{array}$ & $\begin{array}{c}0.00000 \\
(0.00000)\end{array}$ & $\begin{array}{c}0.00000 \\
(0.00000)\end{array}$ & $\begin{array}{c}0.05110 \\
(0.01001)\end{array}$ \\
\hline
\end{tabular}

Note: Each cell contains the average in the first line and the standard deviation in parentheses in the second line based on 100 replications. FNM, false negative measure; FPM, false positive measure; LASSO+rDP, least absolute shrinkage and selection operator with reduced dynamic programming; TLASSO, thresholded least absolute shrinkage and selection operator; WBS, wild binary segmentation.

For the second data generating model, we observe similar comparison results summarized in Table II. In particular, in all noise levels, LASSO+rDP underestimates the number of change points. In all cases, WBS leads to the highest FPM.

In summary, as discussed by Harchaoui and Lévy-Leduc, ${ }^{10}$ LASSO alone is not good for change-point detection. Although WBS performs similarly with the proposed TLASSO-based detection method in terms of FNM in these simulation studies, WBS produces larger FPM in all cases. Such tendency of WBS will generate frequent false positives in practice, which will be seen in our case study in Section 4.

\section{Change-point detection in solar panel degradation}

In this section, the methodology discussed in Section 2 is applied to a case study of performance degradation analysis for solar panel systems. This case study considers detecting abrupt changes in energy conversion efficiencies of four solar energy facilities with data collected over years (see Table III for the description of facilities and data collection period). Each facility has PV and POA measured with the frequency of $15 \mathrm{~min}$. After aggregating 15-min data, we analyze the daily average PV-to-POA ratio. Figure 7(a) shows the daily average PV-to-POA ratios of four facilities over time. The pattern of data highly depends on the facility and season.

Facilities $A$ and $B$ are at the same location; both collecting data for approximately 3 years but using solar panels from different manufacturers. In the two facilities, we observe significantly different degradation patterns. It appears that Facility $A$ used more advanced technology than Facility B, where the decreasing efficiency was fixed in mid-1999.

The performances of panels at Facilities $A$ and $C$ are similar in the sense that the overall efficiency largely oscillates (drop-rise-drop pattern). We note that Facilities $A$ and $C$ use solar panels from the same manufacturer and have the same data collection period from mid-2007 to mid-2012. Facility D data is the largest dataset covering over 10 years. For Facility D, we do not observe the 'drop-rise-drop' pattern. The distinct degradation pattern would be because its panel manufacturer is different from others.

To preprocess data for change-point detection, we first impute missing data by linear interpolation. We then remove the seasonality from the time series using the seasonal decomposition by moving averages. ${ }^{26}$ Figure $7(\mathrm{~b})$ shows the resulting deseasonalized data that we use to detect change points. In the succeeding discussions, we compare four change-point detection methods: WBS, LASSO, LASSO+rDP, and TLASSO. 


\begin{tabular}{|lcccc|}
\hline \multicolumn{2}{|c|}{ Table III. } \\
Facility & Coscription of solar energy facilities and data collection periods \\
\hline A & $21.5^{\circ} \mathrm{N} 158.2^{\circ} \mathrm{W}$ & 20 & 208.4 & Jul-2007 $\sim$ May-2012 (1377 days) \\
B & $21.5^{\circ} \mathrm{N} 158.2^{\circ} \mathrm{W}$ & 20 & 180.0 & Jan-1998 $\sim$ Mar-2001 (1057 days) \\
C & $21.3^{\circ} \mathrm{N} 157.8^{\circ} \mathrm{W}$ & 20 & 153.1 & Apr-2007 $\sim$ May-2012 (1608 days) \\
D & $21.4^{\circ} \mathrm{N} 157.8^{\circ} \mathrm{W}$ & 10 & 180.0 & Aug-1999 $\sim$ Nov-2009 (3536 days) \\
\hline
\end{tabular}

Note: Array tilt (degree from horizontal) and array azimuth (degree from north) present the setups of solar panels.

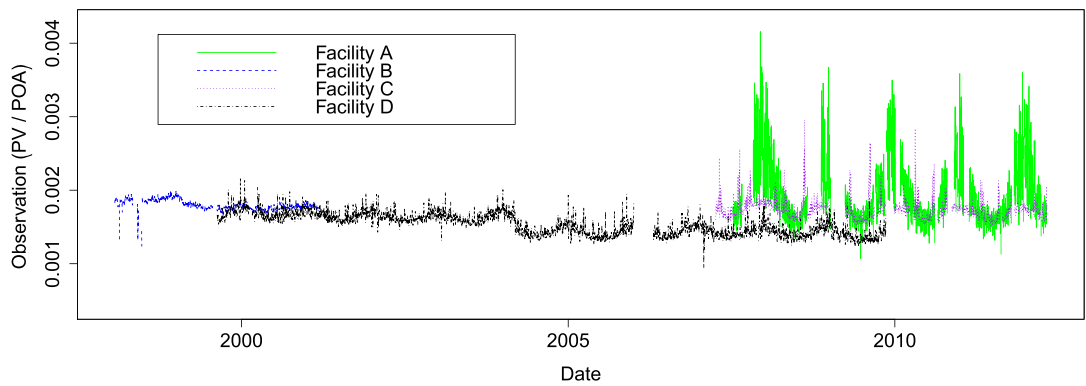

(a) Raw data

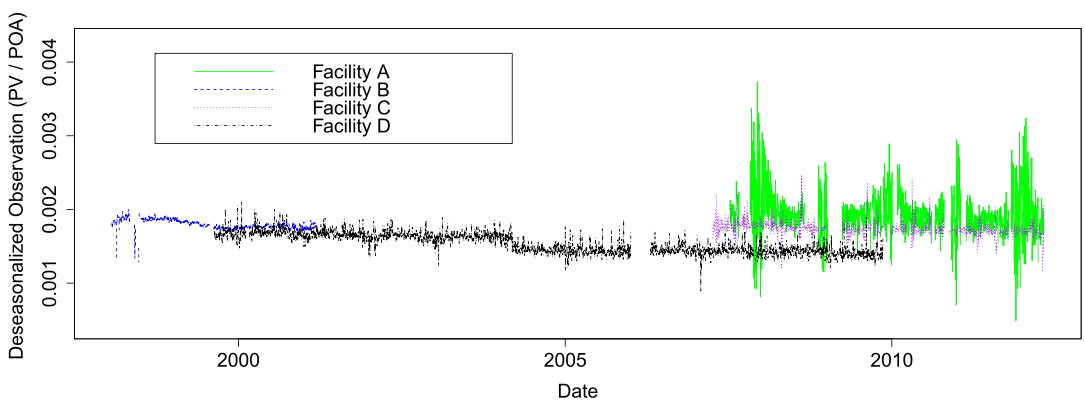

(b) Deseasonalized data

Figure 7. Data plots for four facilities (note the missing data for Facility D in 2006 and for Facilities A and C in 2009)

Table IV summarizes the number of estimated change points from each method for four facilities. Because we do not know the true change points in these datasets, we do not include FPM and FNM in Table IV.

\subsection{Wild binary segmentation detection results}

We use WBS with the number of estimated change points determined by $\mathrm{sSIC}(\alpha=1.01)$, following the recommendation in the study of Fryzlewicz. ${ }^{14}$ The results are as follows:

- Facility A: 35 change points are detected.

- Facility B: 43 change points are detected.

- Facility C: 43 change points are detected.

- Facility D: 50 change points are detected.

Wild binary segmentation suggests more change points than we can possibly observe in real solar panel operations. For example, for Facility D, Figure 8(a) shows the detection result for WBS. The seemingly over-detection results would be because WBS is not designed to guard against false positives but to approximately minimize an estimation error criterion.

From Table IV, we also see that WBS tends to estimate noticeably many change points compared with other three methods.

\subsection{Least absolute shrinkage and selection operator detection results}

The LASSO provides the regularization path that lets us determine how many estimated change points are needed to explain a certain percentage of deviance in the observations. The results are as follows:

- Facility A: 35, 52, and 120 change points account for $10 \%, 20 \%$, and $40 \%$ deviance, respectively.

- Facility B: 5, 19, and 27 change points account for $20 \%, 40 \%$, and $60 \%$ deviance, respectively.

- Facility C: 8, 43, and 108 change points account for $10 \%, 20 \%$, and $40 \%$ deviance, respectively.

- Facility D: Two (2004-03-09 and 2004-03-10) and nine change points account for $20 \%$ and $60 \%$ deviance, respectively. 


\begin{tabular}{|c|c|c|c|c|}
\hline Facility & A & B & C & D \\
\hline WBS & 35 & 43 & 43 & 50 \\
\hline LASSO & 52 & 5 & 43 & 2 \\
\hline LASSO+rDP & 1 & 4 & 1 & 1 \\
\hline TLASSO & 0 & 1 & 0 & 1 \\
\hline
\end{tabular}

Note: LASSO detection results are based on the regularization parameters explaining 20\% deviance. Other methods are based on their recommended settings. LASSO+rDP, least absolute shrinkage and selection operator with reduced dynamic programming; TLASSO, thresholded least absolute shrinkage and selection operator; WBS, wild binary segmentation.

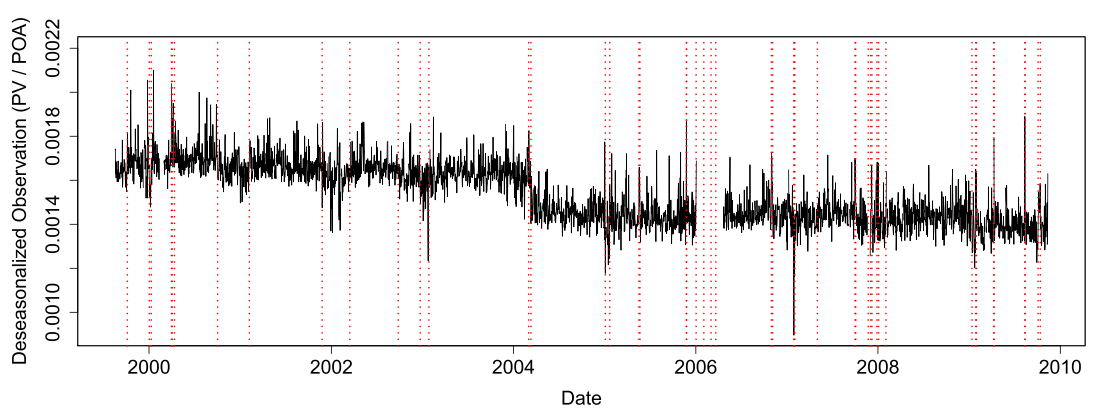

(a) WBS

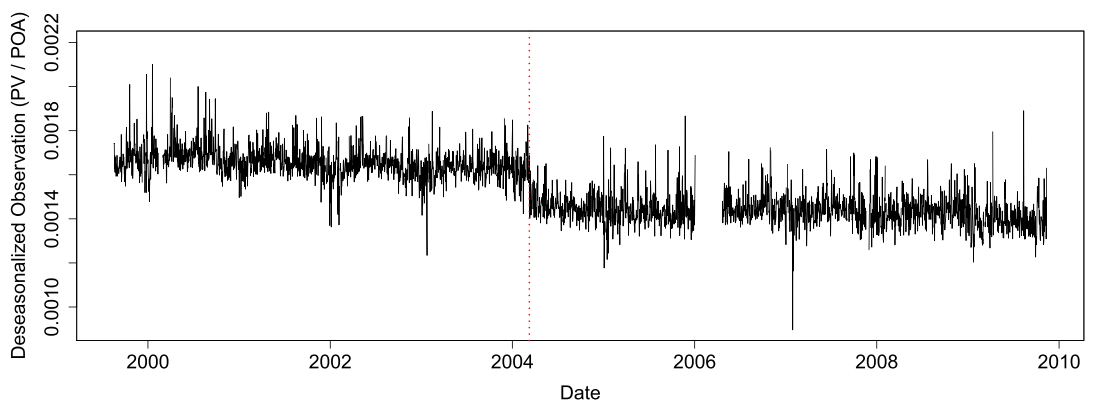

(b) TLASSO

Figure 8. Detection of change points for Facility D by WBS and TLASSO (Note: the red dotted vertical lines indicate the change-point estimates.)

The results indicate that a large number of change points need to be used in order to explain a good amount of deviance (at least $20 \%$ ) in the data. Considering that the significant portion of deviance comes from stochastic noises, it becomes obvious that we need to take an additional step to prune potential false change points.

\subsection{Least absolute shrinkage and selection operator with reduced dynamic programming detection results}

The LASSO+rDP with $v=0.05$ results in a much smaller number of change points than LASSO as follows:

- Facility A: A single change point at 2008-03-23 is detected.

- Facility B: Four change points at 1998-05-31, 1998-06-25, 1999-02-01, and 1999-05-28 are detected.

- Facility C: A single change point at 2008-09-04 is detected.

- Facility D: A single change point at 2004-03-11 is detected.

We can check the plausibility of individual change-point estimates. For example, the change-point estimates for Facilities $\mathrm{A}$ and $\mathrm{C}$ are within the first 9 and 17 months from installation, respectively. Such early change points are unlikely due to actual performance changes, indicating that the method may have chosen at least one change point as an artifact of the method.

\subsection{Thresholded least absolute shrinkage and selection operator detection results}

For TLASSO, we can assume that $\beta_{\min }$ is large for practical reason (i.e., we are interested in abrupt and persistent performance change distinct from stochastic noises). We estimate $\sigma$ by the sample standard deviation of random errors (i.e., the residuals after removing trend and seasonality from the observations). TLASSO results in few or no change points as follows: 
- Facility A: No change point is detected.

- Facility B: A single change point at 1998-06-24 is detected.

- Facility C: No change point is detected.

- Facility D: A single change point at 2004-03-09 is detected.

For Facility B, the change point is located at the date when the solar panel efficiency recovers after noticeable drops, potentially indicating a maintenance activity. For Facility D, Figure 8(b) visually confirms that the solar panel indeed experienced an abrupt performance drop after 4 years from installation.

\section{Conclusion}

Solar energy is a fast growing energy source and has become more and more versatile. Solar energy has been in use for a long time and has allowed the development of efficient, affordable, and easy-to-install solar panels. One of the main challenges in evaluating the performance of solar panel systems is the detection of abrupt changes in energy conversion efficiency. Thus, effective change-point detection in solar panel performance analysis is essential for better harnessing solar energy and making photovoltaic systems more profitable.

In this article, we proposed a nonparametric method for off-line detection of multiple change points in the mean of solar panel's health condition index. Detecting change points via the standard LASSO has low computational complexity but with a major drawback that unnecessarily many change points are chosen, yielding a high false positive rate. We present that TLASSO, originally developed for the parameter estimation and variable selection in a high-dimensional linear model, can be used for abrupt change-point detection. TLASSO helps reduce the false positives while keeping the correctly estimated change points by thresholding the initial estimator obtained from LASSO.

The performance of the TLASSO-based detection method was assessed and compared with benchmark methods using extensive simulations. The simulation concluded that TLASSO is able to accurately detect change points while being robust under many uncertainties. LASSO alone has the worst performance in change-point detection, while additionally pruning by rDP makes LASSO-based detection perform similarly with WBS. TLASSO, however, outperforms them in terms of robustness against false positives while maintaining the similar level of accuracy. To demonstrate how the proposed TLASSO-based detection method can be applied to solar panel analysis, a case study using data collected from four solar energy facilities over years was conducted. Similar to simulation results, the results of case study also indicated that TLASSO outperforms other methods. The proposed method identified physically meaningful change points: one indicating a maintenance activity and the other implying a significant performance drop.

The proposed methodology will be extended to off-line detection of changes with multiple health indexes or signals in future research. Incorporating the findings from change-point detection into solar panel maintenance scheduling, warranty underwriting, cost-benefit analysis, and regulatory policymaking is another important yet challenging topic that deserves further investigation.

\section{Acknowledgements}

This work was partially supported by the National Science Foundation (Grant No. CMMI-1233108, CMMI-1536924). The authors would like to thank Sun Power for Schools Program hosted by Hawaiian Electric Companies, State of Hawaii Department of Education and members of the community for the solar panel installation and data collection. Assistance given by Mr. Steve Luckett from Hawaiian Electric Co Inc. is also greatly appreciated.

\section{References}

1. U.S. Energy Information Administration. Annual energy outlook 2015 with projections to 2040, Technical Report DOE/EIA-0383, U.S. Department of Energy, Washington, DC, 2015.

2. Green Tech Media Research and Solar Energy Industries Association (SEIA). U.S. solar market insight: Year-in-review 2012, Technical Report, San Francisco, CA, 2013.

3. Green Tech Media Research. Global PV demand outlook 2015-2020: Exploring risk in downstream solar markets, Technical Report Boston, MA, 2015.

4. Lombardo T. What is the lifespan of a solar panel?, April 2014. Available from: http://www.engineering.com/ElectronicsDesign/ ElectronicsDesignArticles/ArticleID/7475/What-Is-the-Lifespan-of-a-Solar-Panel.aspx. [Accessed on 15 April 2016].

5. Jordan DC, Kurtz SR. Photovoltaic degradation rates - an analytical review. Progress in Photovoltaics: Research and Applications $2013 ; 21$ (1):12-29.

6. WeatherSpark.com. Historical weather for 2004 in Honolulu, Hawaii, USA. Available from: https://weatherspark.com/history/33125/2004/ Honolulu-Hawaii-United-States. [Accessed on 15 April 2016].

7. U.S. Climate Data. Hawaii. Available from: http://www.usclimatedata.com/climate/hawaii/united-states/3181. [Accessed on 15 April 2016].

8. Roy S, Atchade Y, Michailidis G. Change-point estimation in high-dimensional Markov random field models. arXiv preprint arXiv:1405.6176 2014.

9. Bai J. Estimation of a change point in multiple regression models. Review of Economics and Statistics 1997; 79(4):551-563.

10. Harchaoui Z, Lévy-Leduc C. Multiple change-point estimation with a total variation penalty. Journal of the American Statistical Association 2010; 105(492):1480-1493.

11. Killick R, Fearnhead P, Eckley IA. Optimal detection of changepoints with a linear computational cost. Journal of the American Statistical Association 2012; 107(500):1590-1598.

12. Brodsky BE, Darkhovsky BS. Nonparametric Methods in Change Point Problems. Springer: Dordrecht, 1993.

13. Carlstein EG, Müller HG, Siegmund D. Change-point Problems. IMS Monograph. IMS: Hayward, CA, 1994.

14. Fryzlewicz P. Wild binary segmentation for multiple change-point detection. The Annals of Statistics 2014; 42(6):2243-2281. 
15. Harchaoui Z, Lévy-Leduc C. Catching change-points with lasso, in Advances in Neural Information Processing Systems. MIT Press: Cambridge, MA, 2008; 617-624.

16. Zhou S. Thresholded lasso for high dimensional variable selection and statistical estimation. arXiv preprint arXiv:1002.1583 2010.

17. Choe Y, Guo W, Byon E, Jin J, Li J. Change-point detection in solar panel performance analysis. Proceedings of the 2016 Industrial and Systems Engineering Research Conference, Anaheim, CA, 2016.

18. Efron B, Hastie T, Johnstone I, Tibshirani R. Least angle regression. The Annals of Statistics 2004; 32(2):407-499.

19. Zhao P, Yu B. On model selection consistency of lasso. Journal of Machine Learning Research 2006; 7:2541-2563.

20. Meinshausen N, Yu B. Lasso-type recovery of sparse representations for high-dimensional data. The Annals of Statistics 2009; 37(1):246-270.

21. van de Geer S, Bühlmann P, Zhou S. The adaptive and the thresholded lasso for potentially misspecified models (and a lower bound for the lasso). Electronic Journal of Statistics 2011; 5:688-749.

22. Keener RW. Theoretical Statistics: Topics for a Core Course. Springer-Verlag: New York, 2010.

23. Zou H. The adaptive lasso and its oracle properties. Journal of the American Statistical Association 2006; 101(476):1418-1429.

24. Boysen L, Kempe A, Liebscher V, Munk A, Wittich O. Consistencies and rates of convergence of jump-penalized least squares estimators. The Annals of Statistics 2009; 37(1):157-183.

25. Donoho DL, Johnstone IM. Adapting to unknown smoothness via wavelet shrinkage. Journal of the American Statistical Association 1995; 90(432):1200-1224.

26. Kendall MG, Stuart A. The Advanced Theory of Statistics, Vol. 3. Macmillan: London, 1983.

\section{Authors' biographies}

Youngjun Choe is an Assistant Professor with the Department of Industrial and Systems Engineering at the University of Washington, Seattle. He received his PhD degree in Industrial and Operations Engineering from the University of Michigan, Ann Arbor in 2016. His current research focuses on computational statistics for engineering applications.

Weihong Guo is an Assistant Professor in the Department of Industrial and Systems Engineering at Rutgers University. She received her BS degree in Industrial Engineering from Tsinghua University, China, and her PhD in Industrial and Operations Engineering from the University of Michigan, Ann Arbor. Her research interests are in online process monitoring, data fusion in the interface between applied statistics and system control/optimization, and quality-oriented design and modeling of complex manufacturing systems.

Eunshin Byon received her PhD degree in Industrial and Systems Engineering from Texas A\&M University, College Station, TX, USA, in 2010. She is an Assistant Professor with the Department of Industrial and Operations Engineering, University of Michigan, Ann Arbor, $\mathrm{MI}$, USA. Her research interests include data analytics, quality and reliability engineering, and uncertainty quantification. Prof. Byon is a member of IIE, INFORMS and ASQ.

Jionghua (Judy) Jin is a Professor in the Department of Industrial and Operations Engineering at the University of Michigan. She received her PhD degree from the University of Michigan in 1999. Her recent research focuses on data fusion for improving systems quality and operational efficiency by integrating statistics, signal processing, reliability, systems modeling and decision-making. She has received a number of awards including a NSF CAREER Award in 2002 and a PECASE Award in 2004, and ten Best Paper Awards since 2005. She is a fellow of American Society of Mechanical Engineers (ASME) and Institute of Industrial Engineering (IIE), and an elected senior member of the International Statistical Institute.

Jingjing Li is an Associate Professor in the Harold and Inge Marcus Department of Industrial and Manufacturing Engineering at the Pennsylvania State University. She earned her PhD in Mechanical Engineering in 2011 from the University of Michigan, Ann Arbor. She was selected as an NSF CAREER awardee in 2016. She is a guest editor for the ASME Journal of Manufacturing Science and Engineering, served on NSF panels, organized the 9th International Workshop on Microfactories (IWMF 2014), and has served as symposium chairs for ASME International Manufacturing Science and Engineering Conferences (MSEC) since 2013. Her primary research interest focuses on materials processing, characterization and degradation; and she has published more than 30 peer-reviewed journal papers, including nature communications. 